\title{
Strong Insertion of A Baire-one Function
}

\author{
Majid Mirmiran
}

\author{
Department of Mathematics, University of Isfahan, Isfahan , 81746-73441, Iran \\ mirmir@sci.ui.ac.ir
}

Copyright (C2013 Horizon Research Publishing All rights reserved.

\begin{abstract}
Necessary and sufficient conditions in terms of lower cut sets are given for the strong insertion of a Baire-one function between two comparable realvalued functions on the topological spaces that $\Lambda$-sets are $G_{\delta}-$ sets.
\end{abstract}

Keywords Strong insertion, Strong binary relation, Baire-one function, $\Lambda$-sets, Lower cut set.

\section{Introduction}

A generalized class of closed sets was considered by Maki in 1986 [7]. He investigated the sets that can be represented as union of closed sets and called them $V$-sets. Complements of $V$-sets, i.e., sets that are intersection of open sets are called $\Lambda$-sets [7]. In addition,the insertion of a Baire- one function has also recently considered by the author in [9].

Results of Katětov [3] , [4] concerning binary relations and the concept of an indefinite lower cut set for a real-valued function, which is due to Brooks [2], are used in order to give necessary and sufficient conditions for the strong insertion of a Baire-one function between two comparable real-valued functions on the topological spaces that $\Lambda$-sets are $G_{\delta}$-sets.

A real-valued function $f$ defined on a topological space $X$ is called Baire-one if the preimage of every open subset of $R$ is a $F_{\sigma}$-set in $X$.

If $g$ and $f$ are real-valued functions defined on a space $X$, we write $g \leq f$ in case $g(x) \leq f(x)$ for all $x$ in $X$.

The following definitions are modifications of conditions considered in [5].

A property $P$ defined relative to a real-valued function on a topological space is a $B_{1}$-property provided that any constant function has property $P$ and provided that the sum of a function with property $P$ and any Baire-one function also has property $P$. If $P_{1}$ and $P_{2}$ are $B_{1}$-properties, the following terminology is used: (i) A space $X$ has the weak $B_{1}$-insertion property for $\left(P_{1}, P_{2}\right)$ iff for any functions $g$ and $f$ on $X$ such that $g \leq f, g$ has property $P_{1}$ and $f$ has property $P_{2}$, then there exists a Baire-one function $h$ such that $g \leq h \leq f$. (ii) A space $X$ has the strong $B_{1}$-insertion property for $\left(P_{1}, P_{2}\right)$ iff for any functions $g$ and $f$ on $X$ such that $g \leq f, g$ has property $P_{1}$ and $f$ has property $P_{2}$, then there exists a Baire-one function $h$ such that $g \leq h \leq f$ and such that if $g(x)<f(x)$ for any $\mathrm{x}$ in $\mathrm{X}$, then $g(x)<h(x)<f(x)$.

In this paper, for a topological space that $\Lambda$-sets are $G_{\delta}$-sets, is given a sufficient condition for the weak $B_{1}$-insertion property. Also for a space with the weak $B_{1}$-insertion property, we give necessary and sufficient conditions for the space to have the strong $B_{1}$-insertion property. Several insertion theorems are obtained as corollaries of these results.

\section{The main results}

Before giving a sufficient condition for insertability of a Baire-one function, the necessary definitions and terminology are stated.

Definition. Let $A$ be a subset of a topological space $(X, \tau)$. We define the subsets $A^{\Lambda}$ and $A^{V}$ as follows: $A^{\Lambda}=\cap\{O: O \supseteq A, O \in(X, \tau)\}$ and $A^{V}=\cup\{F: F \subseteq$ $\left.A, F^{c} \in(X, \tau)\right\}$.

$A^{\Lambda}$ is called kernel of $A$.

The following first two definitions are modifications of conditions considered in [3], [4].

Definition. If $\rho$ is a binary relation in a set $S$ then $\bar{\rho}$ is defined as follows: $x \bar{\rho} y$ if and only if $y \rho \nu$ implies $x \rho \nu$ and $u \rho x$ implies $u \rho y$ for any $u$ and $v$ in $S$.

Definition. A binary relation $\rho$ in the power set $P(X)$ of a topological space $X$ is called a strong binary relation in $P(X)$ in case $\rho$ satisfies each of the following conditions:

1) If $A_{i} \rho B_{j}$ for any $i \in\{1, \ldots, m\}$ and for any $j \in$ $\{1, \ldots, n\}$, then there exists a set $C$ in $P(X)$ such that $A_{i} \rho C$ and $C \rho B_{j}$ for any $i \in\{1, \ldots, m\}$ and any $j \in\{1, \ldots, n\}$.

2) If $A \subseteq B$, then $A \bar{\rho} B$.

3) If $A \rho B$, then $A^{\Lambda} \subseteq B$ and $A \subseteq B^{V}$.

The concept of a lower indefinite cut set for a real-valued 
function was defined by Brooks [2] as follows:

Definition. If $f$ is a real-valued function defined on a space $X$ and if $\{x \in X: f(x)<\ell\} \subseteq A(f, \ell) \subseteq\{x \in X$ : $f(x) \leq \ell\}$ for a real number $\ell$, then $A(f, \ell)$ is a lower indefinite cut set in the domain of $f$ at the level $\ell$.

We now give the following main results:

Theorem 2.1. Let $g$ and $f$ be real-valued functions on the topological space $X$, that $\Lambda$-sets in $X$ are $G_{\delta}$-sets, with $g \leq f$. If there exists a strong binary relation $\rho$ on the power set of $X$ and if there exist lower indefinite cut sets $A(f, t)$ and $A(g, t)$ in the domain of $f$ and $g$ at the level $t$ for each rational number $t$ such that if $t_{1}<t_{2}$ then $A\left(f, t_{1}\right) \rho A\left(g, t_{2}\right)$, then there exists a Baire-one function $h$ defined on $X$ such that $g \leq h \leq f$.

Proof. Theorem 2.1, of [9].

If a space has the strong $B_{1}$-insertion property for $\left(P_{1}, P_{2}\right)$, then it has the weak $B_{1}$-insertion property for $\left(P_{1}, P_{2}\right)$.The following result uses lower cut sets and gives a necessary and sufficient condition for a space satisfies that weak $B_{1}$-insertion property to satisfy the strong $B_{1}$-insertion property.

Theorem 2.2. Let $P_{1}$ and $P_{2}$ be $B_{1}$-property and $X$ be a space that satisfies the weak $B_{1}$-insertion property for $\left(P_{1}, P_{2}\right)$. Also assume that $g$ and $f$ are functions on $X$ such that $g \leq f, g$ has property $P_{1}$ and $f$ has property $P_{2}$. The space $X$ has the strong $B_{1}$-insertion property for $\left(P_{1}, P_{2}\right)$ iff there exist lower cut sets $A\left(f-g, 2^{-n}\right)$ and there exists a sequence $\left\{F_{n}\right\}$ of subsets of $X$ such that (i) for each $n, F_{n}$ and $A\left(f-g, 2^{-n}\right)$ are completely separated by Baire-one functions, and (ii) $\{x \in X:(f-g)(x)>$ $0\}=\bigcup_{n=1}^{\infty} F_{n}$.

Proof. Suppose that there is a sequence $\left(A\left(f-g, 2^{-n}\right)\right)$ of lower cut sets for $f-g$ and suppose that there is a sequence $\left(F_{n}\right)$ of subsets of $X$ such that

$$
\{x \in X:(f-g)(x)>0\}=\bigcup_{n=1}^{\infty} F_{n}
$$

and such that for each $n$, there exists a Baire-one function $k_{n}$ on $X$ into $\left[0,2^{-n}\right]$ with $k_{n}=2^{-n}$ on $F_{n}$ and $k_{n}=0$ on $A\left(f-g, 2^{-n}\right)$. The function $k$ from $X$ into $[0,1 / 4]$ which is defined by

$$
k(x)=1 / 4 \sum_{n=1}^{\infty} k_{n}(x)
$$

is a Baire-one function by the Cauchy condition and the properties of Baire-one functions, (1) $k^{-1}(0)=\{x \in$ $X:(f-g)(x)=0\}$ and $(2)$ if $(f-g)(x)>0$ then $k(x)<(f-g)(x):$ In order to verify (1), observe that if $(f-g)(x)=0$, then $x \in A\left(f-g, 2^{-n}\right)$ for each $n$ and hence $k_{n}(x)=0$ for each $n$. Thus $k(x)=0$. Conversely, if $(f-g)(x)>0$, then there exists an $n$ such that $x \in F_{n}$ and hence $k_{n}(x)=2^{-n}$. Thus $k(x) \neq 0$ and this verifies (1). Next, in order to establish (2), note that

$$
\{x \in X:(f-g)(x)=0\}=\bigcap_{n=1}^{\infty} A\left(f-g, 2^{-n}\right)
$$

and that $\left(A\left(f-g, 2^{-n}\right)\right)$ is a decreasing sequence. Thus if $(f-g)(x)>0$ then either $x \notin A(f-g, 1 / 2)$ or there exists a smallest $n$ such that $x \notin A\left(f-g, 2^{-n}\right)$ and $x \in A\left(f-g, 2^{-j}\right)$ for $j=1, \ldots, n-1$.

In the former case,

$k(x)=1 / 4 \sum_{n=1}^{\infty} k_{n}(x) \leq 1 / 4 \sum_{n=1}^{\infty} 2^{-n}<1 / 2 \leq(f-g)(x)$,

and in the latter,

$k(x)=1 / 4 \sum_{j=n}^{\infty} k_{j}(x) \leq 1 / 4 \sum_{j=n}^{\infty} 2^{-j}<2^{-n} \leq(f-g)(x)$.

Thus $0 \leq k \leq f-g$ and if $(f-g)(x)>0$ then $(f-g)(x)>$ $k(x)>0$. Let $g_{1}=g+(1 / 4) k$ and $f_{1}=f-(1 / 4) k$. Then $g \leq g_{1} \leq f_{1} \leq f$ and if $g(x)<f(x)$ then

$$
g(x)<g_{1}(x)<f_{1}(x)<f(x) .
$$

Since $P_{1}$ and $P_{2}$ are $B_{1}$-properties, then $g_{1}$ has property $P_{1}$ and $f_{1}$ has property $P_{2}$. Since by hypothesis $X$ has the weak $B_{1}$-insertion property for $\left(P_{1}, P_{2}\right)$ , then there exists a Baire-one function $h$ such that $g_{1} \leq h \leq f_{1}$. Thus $g \leq h \leq f$ and if $g(x)<f(x)$ then $g(x)<h(x)<f(x)$. Therefore $X$ has the strong $B_{1}$-insertion property for $\left(P_{1}, P_{2}\right)$. (The technique of this proof is by Lane [5].)

Conversely, assume that $X$ satisfies the strong $B_{1}$-insertion for $\left(P_{1}, P_{2}\right)$. Let $g$ and $f$ be functions on $X$ satisfying $P_{1}$ and $P_{2}$ respectively such that $g \leq f$. Thus there exists a Baire-one function $h$ such that $g \leq h \leq f$ and such that if $g(x)<f(x)$ for any $x$ in $X$, then $g(x)<h(x)<f(x)$. We follow an idea contained in Powderly [10]. Now consider the functions 0 and $f-h .0$ satisfies property $P_{1}$ and $f-h$ satisfies property $P_{2}$. Thus there exists a Baire-one function $h_{1}$ such that $0 \leq h_{1} \leq f-h$ and if $0<(f-h)(x)$ for any $x$ in $X$, then $0<h_{1}(x)<(f-h)(x)$. We next show that

$$
\{x \in X:(f-g)(x)>0\}=\left\{x \in X: h_{1}(x)>0\right\} .
$$

If $x$ is such that $(f-g)(x)>0$, then $g(x)<f(x)$. Therefore $g(x)<h(x)<f(x)$. Thus $f(x)-h(x)>0$ or $(f-h)(x)>0$. Hence $h_{1}(x)>0$. On the other hand, if $h_{1}(x)>0$, then since $(f-h) \geq h_{1}$ and $f-g \geq f-h$, therefore $(f-g)(x)>0$. For each $n$, let

$$
\begin{aligned}
& A\left(f-g, 2^{-n}\right)=\left\{x \in X:(f-g)(x) \leq 2^{-n}\right\}, \\
& \mathrm{F}_{n}=\left\{x \in X: h_{1}(x) \geq 2^{-n+1}\right\} \text { and } \\
& k_{n}=\sup \left\{\inf \left\{h_{1}, 2^{-n+1}\right\}, 2^{-n}\right\}-2^{-n} .
\end{aligned}
$$

Since $\{\mathrm{x} \in X:(f-g)(x)>0\}=\left\{x \in X: h_{1}(x)>0\right\}$, it follows that

$$
\{x \in X:(f-g)(x)>0\}=\bigcup_{n=1}^{\infty} F_{n} .
$$

We next show that $k_{n}$ is a Baire-one function which completely separates $F_{n}$ and $A\left(f-g, 2^{-n}\right)$. From its definition and by the properties of Baire-one functions, it is clear that $k_{n}$ is a Baire-one function. Let $x \in F_{n}$. Then, from the definition of $k_{n}, k_{n}(x)=2^{-n}$. If $x \in A\left(f-g, 2^{-n}\right)$, then since $h_{1} \leq f-h \leq f-g, h_{1}(x) \leq 2^{-n}$. Thus $k_{n}(x)=0$, according to the definition of $k_{n}$. Hence $k_{n}$ completely 
separates $F_{n}$ and $A\left(f-g, 2^{-n}\right)$.

Theorem 2.3. Let $P_{1}$ and $P_{2}$ be $B_{1}$-properties and assume that the space $X$ satisfied the weak $B_{1}$-insertion property for $\left(P_{1}, P_{2}\right)$. The space $X$ satisfies the strong $B_{1}$-insertion property for $\left(P_{1}, P_{2}\right)$ iff $X$ satisfies the strong $B_{1}$-insertion property for $\left(P_{1}, B_{1}\right)$ and for $\left(B_{1}, P_{2}\right)$.

Proof. Assume that $X$ satisfies the strong $B_{1}$-insertion property for $\left(P_{1}, B_{1}\right)$ and for $\left(B_{1}, P_{2}\right)$. If $g$ and $f$ are functions on $X$ such that $g \leq f, g$ satisfies property $P_{1}$, and $f$ satisfies property $P_{2}$, then since $X$ satisfies the weak $B_{1}$-insertion property for $\left(P_{1}, P_{2}\right)$ there is a Baire-one function $k$ such that $g \leq k \leq f$. Also, by hypothesis there exist Baire-one functions $h_{1}$ and $h_{2}$ such that $g \leq h_{1} \leq k$ and if $g(x)<k(x)$ then $g(x)<h_{1}(x)<k(x)$ and such that $k \leq h_{2} \leq f$ and if $k(x)<f(x)$ then $k(x)<h_{2}(x)<f(x)$. If a function $h$ is defined by $h(x)=\left(h_{2}(x)+h_{1}(x)\right) / 2$, then $h$ is a Baire-one function, $g \leq h \leq f$, and if $g(x)<f(x)$ then $g(x)<h(x)<f(x)$. Hence $X$ satisfies the strong $B_{1}$-insertion property for $\left(P_{1}, P_{2}\right)$. The converse is obvious since any Baire-one function must satisfy both properties $P_{1}$ and $P_{2}$. (The technique of this proof is by Lane [6].)

\subsection{Applications}

Definition. A real-valued function $f$ defined on a space $X$ is called upper semi-Baire-one (resp. lower semiBaire-one) if $f^{-1}(-\infty, t)$ (resp. $\left.f^{-1}(t,+\infty)\right)$ is a $F_{\sigma}$-set for any real number $t$.

The abbreviations $u s c, l s c, u s B_{1}$ and $l s B_{1}$ are used for upper semicontinuous, lower semicontinuous, upper semi-Baire-one, and lower semi-Baire-one, respectively.

Example : Let $X$ be linearly ordered by a relation $\leq$.Take as open subsets for a topology on $X$ all sets of the form $\{x \in X: x<a\}$, for $a \in X$, then $X$ is a topological space that $\Lambda$-sets are $G_{\delta}$-sets.

Note : If $X$ is a $T_{1}$ space, the results of this paper concerning insertion of Baire-one functions are trivial: Every subset of $X$ is a $V$-set since it is a union of singletons.In a space where every $V$-set is an $F_{\sigma}$-set, every subset of $X$ is both an $F_{\sigma}$-set and a $G_{\delta}$-set.For such a space,every real-valued function on $X$ is a Baire-one function.Also, if $X$ is a $T_{1}$ space, for any subset $A$ of $X, A^{\Lambda}$ and $A^{V}$ are both equal to $A$.

Remark 1. [3], [4]. A space $X$ has the weak $c$-insertion property for $(u s c, l s c)$ iff $X$ is normal.

Before stating the consequences of Theorems 2.1, 2.2, and 2.3 we suppose that $X$ is a topological space that $\Lambda$-sets are $G_{\delta}$-sets.

Corollary 3.1. For each pair of disjoint $G_{\delta}$-sets $G_{1}, G_{2}$, there are two $F_{\sigma}$-sets $F_{1}$ and $F_{2}$ such that $G_{1} \subseteq F_{1}, G_{2} \subseteq F_{2}$ and $F_{1} \cap F_{2}=\emptyset$ iff $\mathrm{X}$ has the weak $B_{1}$-insertion property for $\left(u s B_{1}, l s B_{1}\right)$.

Proof. Corollary 3.1, of [9].
Before stating the consequences of Theorem 2.2, we state and prove the necessary lemmas.

Lemma 3.2. The following conditions on the space $X$ are equivalent:

(i) Every two disjoint $G_{\delta}$-sets of $X$ can be separated by $F_{\sigma}$-sets of $X$.

(ii) If $G$ is a $G_{\delta}$-set of $X$ which is contained in a $F_{\sigma}$-set $F$, then there exists a $F_{\sigma}$-set $H$ such that $G \subseteq H \subseteq H^{\Lambda} \subseteq F$.

Proof. Lemma 3.2, of [9].

Lemma 3.3. Suppose that $X$ is the topological space s.t. we can separate every two disjoint $G_{\delta}$-sets by $F_{\sigma}$-sets. If $G_{1}$ and $G_{2}$ are two disjoint $G_{\delta}$-sets of $X$, then there exists a Baire-one function $h$ on $X$ into $[0,1]$ s.t. $h\left(G_{1}\right)=\{0\}$ and $h\left(G_{2}\right)=\{1\}$.

Proof. Lemma 3.3, of [9].

Lemma 3.4. Suppose that $X$ is the topological space s.t. we can separate every two disjoint $G_{\delta}$-sets by $F_{\sigma}$-sets. If $G_{1}$ and $G_{2}$ are two disjoint $G_{\delta}$-sets of $X$ and $G_{1}$ is a countable intersection of $F_{\sigma}$-sets, then there exists a Baire-one function $h$ on $X$ into $[0,1]$ s.t. $h^{-1}(0)=G_{1}$ and $h\left(G_{2}\right)=\{1\}$.

Proof. Suppose that $G_{1}=\bigcap_{n=1}^{\infty} F_{n}$, where $F_{n}$ is a $F_{\sigma}$-set of $X$. We can suppose that $F_{n} \cap G_{2}=\emptyset$, otherwise we can substitute $F_{n}$ by $F_{n} \backslash G_{2}$. By Lemma 3.3, for every $n \in N$, there exists a Baire-one function $h_{n}$ on $X$ into $[0,1]$ s.t. $h_{n}\left(G_{1}\right)=\{0\}$ and $h_{n}\left(X \backslash F_{n}\right)=\{1\}$. We set $h(x)=\sum_{n=1}^{\infty} 2^{-n} h_{n}(x)$.

Since the above series is uniformly convergent, it follows that $h$ is a Baire-one function from $X$ to $[0,1]$. Since for every $n \in N, G_{2} \subseteq X \backslash F_{n}$, therefore $h_{n}\left(G_{2}\right)=\{1\}$ and consequently $h\left(G_{2}\right)=\{1\}$. Since $h_{n}\left(G_{1}\right)=\{0\}$, hence $h\left(G_{1}\right)=\{0\}$. It suffices to show that if $x \notin G_{1}$, then $h(x) \neq 0$.

Now if $x \notin G_{1}$, since $G_{1}=\bigcap_{n=1}^{\infty} F_{n}$, therefore there exists $n_{0} \in N$ s.t. $x \notin F_{n_{0}}$, hence $h_{n_{0}}(x)=1$, i.e., $h(x)>0$. Therefore $h^{-1}(0)=G_{1}$.

Lemma 3.5. Suppose that $X$ is the topological space s.t. we can separate every two disjoint $G_{\delta}$-sets by $F_{\sigma}$-sets. The following conditions are equivalent:

(i) For every two disjoint $G_{\delta}-$ sets $G_{1}$ and $G_{2}$, there exists a Baire-one function $h$ on $X$ into $[0,1]$ s.t. $h^{-1}(0)=G_{1}$ and $h^{-1}(1)=G_{2}$.

(ii) Every $G_{\delta}-$ set is a countable intersection of $F_{\sigma}$-set.

(iii) Every $F_{\sigma}$-set is a countable union of $G_{\delta}$-set.

Proof. (i) $\Rightarrow$ (ii). Suppose that $G$ is a $G_{\delta}-$ sets. Since $\emptyset$ is a $G_{\delta}$-set, by (i) there exists a Baire-one function $h$ on $X$ into $[0,1]$ s.t. $h^{-1}(0)=G$. Set $F_{n}=\{x \in X$ : $\left.h(x)<\frac{1}{n}\right\}$. Then for every $n \in N, F_{n}$ is a $F_{\sigma}-$ set and $\bigcap_{n=1}^{\infty} F_{n}=\{x \in X: h(x)=0\}=G$.

(ii) $\Rightarrow$ (i). Suppose that $G_{1}$ and $G_{2}$ are two disjoint $G_{\delta}$-sets. By Lemma 3.4, there exists a Baireone function $f$ on $X$ into $[0,1]$ s.t. $f^{-1}(0)=G_{1}$ and $f\left(G_{2}\right)=\{1\}$. Set $F=\left\{x \in X: f(x)<\frac{1}{2}\right\}, G=\{x \in$ $\left.X: f(x)=\frac{1}{2}\right\}$, and $H=\left\{x \in X: f(x)>\frac{1}{2}\right\}$. Then $F \cup G$ and $H \cup G$ are two $G_{\delta}$-sets and $(F \cup G) \cap G_{2}=\emptyset$. By Lemma 3.4, there exists a Baire-one function $g$ on $X$ 
into $\left[\frac{1}{2}, 1\right]$ s.t. $g^{-1}(1)=G_{2}$ and $g(F \cup G)=\left\{\frac{1}{2}\right\}$. Define $h$ by $h(x)=f(x)$ for $x \in F \cup G$, and $h(x)=g(x)$ for $x \in H \cup G . h$ is well-defined and a Baire-one function, since $(F \cup G) \cap(H \cup G)=G$ and for every $x \in G$ we have $f(x)=g(x)=\frac{1}{2}$. Furthermore, $(F \cup G) \cup(H \cup G)=X$, hence $h$ defined on $X$ and maps to $[0,1]$. Also, we have $h^{-1}(0)=G_{1}$ and $h^{-1}(1)=G_{2}$.

(ii) $\Leftrightarrow$ (iii) By De Morgan law and noting that the complement of every $G_{\delta}$-set is a $F_{\sigma}$-set and complement of every $F_{\sigma}$-set is a $G_{\delta}$-set, the equivalence is hold.

Remark 2. [8] . A space $X$ has the strong $c$-insertion property for $(u s c, l s c)$ iff $X$ is perfectly normal.

Corollary 3.6. For every two disjoint $G_{\delta}-$ sets $G_{1}$ and $G_{2}$, there exists a Baire-one function $h$ on $X$ into $[0,1]$ s.t. $h^{-1}(0)=G_{1}$ and $h^{-1}(1)=G_{2}$ iff $X$ has the strong $B_{1}$-insertion property for $\left(u s B_{1}, l s B_{1}\right)$.

Proof. Since for every two disjoint $G_{\delta}-$ sets $G_{1}$ and $G_{2}$, there exists a Baire-one function $h$ on $X$ into $[0,1]$ s.t. $h^{-1}(0)=G_{1}$ and $h^{-1}(1)=G_{2}$, define $F_{1}=\{x \in$ $\left.X: h(x)<\frac{1}{2}\right\}$ and $F_{2}=\left\{x \in X: h(x)>\frac{1}{2}\right\}$. Then $F_{1}$ and $F_{2}$ are two disjoint $F_{\sigma}$-sets that contain $G_{1}$ and $G_{2}$, respectively. This means that, we can separate every two disjoint $G_{\delta}$-sets by $F_{\sigma}$-sets. Hence by Corollary 3.1, $X$ has the weak $B_{1}$-insertion property for $\left(u s B_{1}, l s B_{1}\right)$. Now, assume that $g$ and $f$ are functions on $X$ such that $g \leq f, g$ is $u s B_{1}$ and $f$ is $l s B_{1}$. Since $f-g$ is $l s B_{1}$, therefore the lower cut set $A\left(f-g, 2^{-n}\right)=\{x \in X$ : $\left.(f-g)(x) \leq 2^{-n}\right\}$ is a $G_{\delta}$-set. By Lemma 3.5, we can choose a sequence $\left\{G_{n}\right\}$ of $G_{\delta}$-sets s.t. $\{x \in X$ : $(f-g)(x)>0\}=\bigcup_{n=1}^{\infty} G_{n}$ and for every $n \in N, G_{n}$ and $A\left(f-g, 2^{-n}\right)$ are disjoint. By Lemma 3.3, $G_{n}$ and $A\left(f-g, 2^{-n}\right)$ can be completely separated by Baire-one functions. Hence by Theorem 2.2, $X$ has the strong $B_{1}$-insertion property for $\left(u s B_{1}, l s B_{1}\right)$.

On the other hand, suppose that $G_{1}$ and $G_{2}$ are two disjoint $G_{\delta}$-sets. Since $G_{1} \cap G_{2}=\emptyset$, hence $G_{2} \subseteq G_{1}^{c}$. Set $g=\chi_{G_{2}}$ and $f=\chi_{G_{1}^{c}}$. Then $f$ is $l s B_{1}$ and $g$ is $u s B_{1}$ and furthermore $g \leq f$. By hypothesis, there exists a Baire-one function $h$ on $X$ s.t. $g \leq h \leq f$ and whenever $g(x)<f(x)$ we have $g(x)<h(x)<f(x)$. By definitions of $f$ and $g$, we have $h^{-1}(1)=G_{2} \cap G_{1}^{c}=G_{2}$ and $h^{-1}(0)=G_{1} \cap G_{2}^{c}=G_{1}$.

Remark 3. [11]. A space $X$ has the weak $c$-insertion property for $(l s c, u s c)$ iff $X$ is extremally disconnected.

Corollary 3.7. For every $F$ of $F_{\sigma}$-set, $F^{\Lambda}$ is a $F_{\sigma}$-set iff $X$ has the weak $B_{1}$-insertion property for $\left(l s B_{1}, u s B_{1}\right)$.

Proof. Corollary 3.6, of [9].

Remark 4. [1]. A space $X$ has the strong $c$-insertion property for $(l s c, u s c)$ iff each open subset of $X$ is closed.

Corollary 3.8. Every $F_{\sigma}$-set is a $G_{\delta}$-set iff $X$ has the strong $B_{1}$-insertion property for $\left(l s B_{1}, u s B_{1}\right)$.

Proof. By hypothesis, for every $F$ of $F_{\sigma}$-set, we have $F^{\Lambda}=F$ is a $F_{\sigma}$-set. Hence by Corollary $3.7, X$ has the weak $B_{1}$-insertion property for $\left(l s B_{1}, u s B_{1}\right)$. Now, assume that $g$ and $f$ are functions on $X$ such that $g \leq f, g$ is $l s B_{1}$ and $f$ is $B_{1}$. Set $A\left(f-g, 2^{-n}\right)=$ $\left\{x \in X:(f-g)(x)<2^{-n}\right\}$. Then, since $f-g$ is $u s B_{1}$, we can say that $A\left(f-g, 2^{-n}\right)$ is a $F_{\sigma}$-set. By hypothesis, $A\left(f-g, 2^{-n}\right)$ is a $G_{\delta}-$ set. Set $F_{n}=$ $X \backslash A\left(f-g, 2^{-n}\right)$. Then $F_{n}$ is a $F_{\sigma}$-set. This means that $F_{n}$ and $A\left(f-g, 2^{-n}\right)$ are disjoint $F_{\sigma}$-sets and also are two disjoint $G_{\delta}$-sets. Therefore $F_{n}$ and $A\left(f-g, 2^{-n}\right)$ can be completely separated by Baire-one functions. Now, we have $\bigcup_{n=1}^{\infty} F_{n}=\{x \in X:(f-g)(x)>0\}$. By Theorem 2.2, $X$ has the strong $B_{1}$-insertion property for $\left(l s B_{1}, B_{1}\right)$. By an analogous argument, we can prove that $X$ has the strong $B_{1}$-insertion property for $\left(B_{1}, u s B_{1}\right)$. Hence, by Theorem $2.3, X$ has the strong $B_{1}$-insertion property for $\left(l s B_{1}, u s B_{1}\right)$.

On the other hand, suppose that $X$ has the strong $B_{1}$-insertion property for $\left(l s B_{1}, u s B_{1}\right)$. Also, suppose that $F$ is a $F_{\sigma}$-set. Set $f=1$ and $g=\chi_{F}$. Then $f$ is $u s B_{1}, g$ is $l s B_{1}$ and $g \leq f$. By hypothesis, there exists a Baire-one function $h$ on $X$ s.t. $g \leq h \leq f$ and whenever $g(x)<f(x)$, we have $g(x)<h(x)<f(x)$. It is clear that $h(F)=\{1\}$ and for $x \in X \backslash F$ we have $0<h(x)<1$. Since $h$ is a Baire-one function, therefore $\{x \in X: h(x) \geq 1\}=F$ is a $G_{\delta}$-set, i.e., $F$ is a $G_{\delta}-$ set.

\section{Acknowledgements}

This research was partially supported by Centre of Excellence for Mathematics(University of Isfahan).

\section{REFERENCES}

[1] J. Blatter and G. L. Seever, Interposition of semicontinuous functions by continuous functions, Analyse Fonctionelle et Applications (Comptes Rendus du colloque d' Analyse, Rio de Janeiro 1972), Hermann, Paris, 1975, 27-51.

[2] F. Brooks, Indefinite cut sets for real functions, Amer. Math. Monthly, 78(1971), 1007-1010.

[3] M. Katětov, On real-valued functions in topological spaces, Fund. Math., 38(1951), 85-91.

[4] M. Katětov, Correction to, "On real-valued functions in topological spaces", Fund. Math., 40(1953), 203-205.

[5] E. Lane, Insertion of a continuous function, Pacific J. Math., 66(1976), 181-190.

[6] E. Lane, PM-normality and the insertion of a continuous function, Pacific J. of Math., 82(1979), 155162.

[7] H. Maki, Generalized $\Lambda$-sets and the associated closure operator, The special Issue in commemoration of Prof. Kazuada IKEDA's Retirement, (1986), 139-146.

[8] E. Michael, Continuous selections I, Ann. of Math., 63(1956), 361-382. 
[9] M. Mirmiran, Insertion of a Baire-one function, Mathematics and Statistics, Vol. 1, No. 1 (2013), 5-9.

[10] M. Powderly, On insertion of a continuous function, Proceedings of the A.M.S., 81(1981), 119-120.
[11] M. H. Stone, Boundedness properties in functionlattices, Canad. J. Math., 1(1949), 176-189. 